\title{
Role of Intensity Modulated Radiotherapy (IMRT) in the Treatment of High- Grade Gliomas
}

\author{
Abdalla Mohammed Hassan Emam, ${ }^{1}$ MSc, Wael El-sheshtawy, ${ }^{1}$ MD, Ebrahim Ewais, ${ }^{2}$ MD, Hassan \\ Hamdy, ${ }^{1}$ MD.
}

\author{
* Corresponding Author: \\ Abdalla Mohammed Hassan \\ Emam \\ drabdallaemam@gmail.com
}

Received for publication September 27, 2020; Accepted December 20, 2020; Published online December 20, 2020.

\section{Copyright 2020 The Authors} published by Al-Azhar University, Faculty of Medicine, Cairo, Egypt. All rights reserved. This an open-access article distributed under the legal terms, where it is permissible to download and share the work provided it is properly cited. The work cannot be changed in any way or used commercially.

\section{doi: 10.21608/aimj.2020.43502.1325.}

${ }^{l}$ Clinical Oncology and Nuclear Medicine Department, Faculty of Medicine, Al-Azhar University.

${ }^{2}$ Neurosurgery Department, Faculty of Medicine, Al-Azhar University

\begin{abstract}
Background: High-grade gliomas (HGG) represent the commonest primary adult brain tumors. Radiotherapy plays an important role in the management of this disease.

Aim of the study: To assess the patterns of failure, treatment-related toxicities, progression-free survival (PFS), and overall survival (OS), following limited-margins.

Patients and Methods: In this prospective phase II study, patients with HGG have been treated with IMRT using the ESTRO-ACROP contouring guidelines that not include the peritumoral edema in the gross target volume (GTV) with adding $2 \mathrm{~cm}$ margin around the GTV to create the clinical target volume (CTV), in one phase, after maximum safe resection of the tumor or stereotactic biopsy.

Results: The trial included 40 patients with a median age of 49 years, 16 patients $(40 \%)$ were in complete remission, 13 patients $(12.5 \%)$ had partial regression, and stable disease occurred in 11 patients $(27.5 \%)$. The progression/failure occurred in 28 patients $(70 \%)$. Failures were located infield in 24 patients (86\%), out-field in two patients (7\%), and were marginal in two cases (7\%). No notable serious toxicities during the treatment course; the commonest toxicities were G1-2 headache and vomiting $( \pm 72.5 \%)$. After a median follows up of 12.5 months; the median PFS and OS were 11.7 and 14.3 months respectively.

Conclusion: In this study, the majority of relapses following limited volume IMRT occurred within the field of radiotherapy with acceptable treatment-related toxicities and oncological outcomes, which suggest the use of IMRT with limited CTV as an acceptable treatment technique in HGG patients
\end{abstract}

Keywords: IMRT; High-Grade Gliomas; limited volumes. spinal.

Disclosure: The authors have no financial interest to declare in relation to the content of this article. The Article Processing Charge was paid for by the authors.

Authorship: All authors have a substantial contribution to the article.

\section{INTRODUCTION}

The standard approach for the treatment of high-grade gliomas (HGGs) is the combination of surgery, radiotherapy, and temozolomide in selected patients. ${ }^{1}$

Intensity Modulated Radiotherapy (IMRT) is a widely used treatment technique for HGGs which provides high dose conformity; better normal tissue sparing and less neurotoxicity. However, optimal RT treatment volumes remain controversial. ${ }^{2}$

The Radiation Therapy Oncology Group (RTOG) recommends the initial CTV include the postoperative Magnetic Resonance Images (MRI) T2weighted (enhance tumor and peritumoral edema) plus a $2 \mathrm{~cm}$ margin, followed by a boost field for the residual enhanced tumor on $\mathrm{T} 1$ sequence and the surgical cavity plus $2.5 \mathrm{~cm}^{3}$

The European Organization for Research and Treatment of Cancer (EORTC) favors a single-phase technique, consisting of $60 \mathrm{~Gy}$ over 30 fractions. The GTV is defined as the region of enhancement (without edema) on preoperative MRI for patients who underwent biopsy or the surgical tumor bed plus any residual enhancing tumor for patients who underwent resection. The CTV is defined as the GTV plus two $\mathrm{cm}$ which is modified in anatomic regions. ${ }^{4}$

The Adult Brain Tumor Consortium (ABTC) recommends delivering $60 \mathrm{~Gy}$ in two phases. The initial CTV is $5 \mathrm{~mm}$ around the tumor and surrounding 
edema on postoperative T2 or FLAIR MRI which expanded by $0.5 \mathrm{~cm}$ to create the initial The planning target volume (PTV). The boost GTV includes the residual $\mathrm{T} 1$ contrast-enhancing tumor plus resection cavity and expanded similarly. ${ }^{5}$

Because most of the recurrences are located within $2 \mathrm{~cm}$ from the gross residual tumor according to many reports. ${ }^{5-9}$ We have adopted in this study the EORTC delineation guidelines and used the IMRT technique to minimize the radiotherapy related toxicity.

The study aimed to assess the patterns of failure in relation to $\mathrm{CTV}$, treatment-related toxicities, response rate; PFS, and OS following limited-margins IMRT according to the EORTC-ACROP guidelines.

\section{PATIENTS AND METHODS}

In this prospective phase II study; newly diagnosed adult patients with HGGs, and performance status (ECOG PS) 0-2, were included after recovery from the surgical procedures. Computed tomography $(\mathrm{CT})$ was obtained in the treatment position using a thermoplastic mask for fixation. The CT was fused with postoperative gadolinium-enhanced MRI images, and then the normal structures and tumor volumes were contoured on the fused images.

The Surgical tumor bed with any residual enhancing tumor seen in the postoperative contrast-enhanced T1weighted MRI was considered GTV. The CTV was created by adding a $2 \mathrm{~cm}$ and modified anatomically where natural barriers exist. The peritumoral edema was not intentionally included within CTV. The PTV was defined by adding $0.5 \mathrm{~cm}$ to CTV. The radiotherapy was delivered using the IMRT technique in one phase conventional dosing schedule (60Gy in 30 fractions over 6 weeks). The dose was lowered to $54 \mathrm{~Gy}$ when tolerance of organs at risk (OAR) was not achieved.

Conformity index $(\mathrm{CI})$ is defined as a ratio between the volume covered by the reference isodose $(95 \%)$ and the total volume of PTV; ideally $=1$, calculated from the equation $\mathrm{CI}_{\mathrm{RTOG}}=\mathrm{V}_{\mathrm{RI}} / \mathrm{TV}$; where $\mathrm{V}_{\mathrm{RI}}=$ Reference isodose volume and $\mathrm{TV}=$ Target volume While Homogeneity index (HI) was calculated by the following formula; $\mathrm{HI}=\mathrm{D}_{5} / \mathrm{D}_{95}$; where $\mathrm{D}_{5}=$ minimum dose in $5 \%$ of $\mathrm{TV}$ and $\mathrm{D}_{95}=$ minimum dose in $95 \%$ of $\mathrm{TV}$; ideally $=1$. $^{10}$

Temozolomide was given in a dose of $75 \mathrm{mg} / \mathrm{m}^{2}$ during the whole course of RT, while adjuvant temozolomide was used only for GBM patients, in a dose of $150 \mathrm{mg} / \mathrm{m}^{2} / \mathrm{d}$ for five days, every 28 days.

Evaluation of response was done according to the Updated Response Assessment in Neuro-Oncology (RANO) criteria for high-grade gliomas. ${ }^{11}$ Relapses have been classified into 3 types based on the site of recurrence in relation to the CTV as following, infield recurrence (within 95\% isodose curve), marginal recurrence (crosses 95\% isodose curve), and outfield recurrence (completely outside $95 \%$ isodose curve) ${ }^{8}$.

\section{Statistical analysis:}

Data were analyzed using IBM SPSS (Statistical Package for Social Sciences), version 24. Numerical data were described as median and range or mean and standard deviation (SD) as appropriate, while qualitative data were described as number and percentage. Survival analysis was done using the Kaplan-Meier method. Multivariate analysis was done by the Cox regression model to test for the independent prognostic effect of statistically significant variables on the univariate level with calculating the hazard ratio and its $95 \%$ confidence interval. A p-value less than or equal to 0.05 was considered statistically significant.

\section{RESULTS}

For the included 40 patients; The median age was 49 years $(18-68$ years $)$, male to female percentage was 2:1, 24 patients $(60.0 \%)$ were presented with ECOG PS 1, while 16 patients (40\%) with ECOG PS II. (Table 1)

Manifestations of increased intracranial tension (ICT) were the most common presenting symptoms that were seen in 32 patients (80\%), motor weakness was manifested in 15 patients $(37.5 \%)$, and convulsions in 3 patients (7.5\%). Twenty-three patients $(57.5 \%)$ presenting by tumors $\geq 5 \mathrm{~cm}$. (Table 1 )

Thirty-six patients (90\%) underwent surgical resection and four patients $(10 \%)$ were subjected to stereotactic biopsy. The mean diameter of the postoperative residual tumor was $4.81 \mathrm{~cm}$ (SD: 1.53 ). (Table 1)

Thirty-four patients $(85 \%)$ received $60 \mathrm{~Gy}$ in 30 fractions and 6 patients $(15 \%)$ received 54Gy in 27 fractions because of the proximity of the tumor to critical structures. The mean overall radiotherapy duration was 45.35 days (SD: 6.64). The median GTV volume was $68.7 \mathrm{~cm}^{3}$ (range: $7.10 \mathrm{~cm}^{3}-379.8 \mathrm{~cm}^{3}$ ). The median CTV volume was $259.8 \mathrm{~cm}^{3}$ (range: $69.8 \mathrm{~cm}^{3}-445 \mathrm{~cm}^{3}$ ) with a mean dose $98.6 \%$ (SD: 2.61 ), the median PTV volume was $348 \mathrm{~cm}^{3}$ (range: $129.6 \mathrm{~cm}^{3}-507 \mathrm{~cm}^{3}$ ) with a mean dose $99.91 \%$ (SD: 1.88). The median conformity index was 0.97 (range: $0.94-1)$ and the median homogeneity index was 1.05 (range: 1.025-1.4).

Concurrent temozolomide was given to 36 patients (90\%); 32 patients of them continue concurrent temozolomide along the whole course of radiotherapy without the need for dose modification and the median daily dose of temozolomide was $100 \mathrm{mg}$. The commonest treatment-related toxicities were G1-2 headache and Vomiting $(72.5 \%)$ with variable degrees, while the deterioration in ECOG PS, convulsions, G1-2 infection and deep venous thrombosis (DVT) were developed in 30\%, 12.5\%, 
$5 \%$, and $2.5 \%$ respectively, with no recorded treatment-related mortality.

\begin{tabular}{|c|c|c|}
\hline & Number & $\%$ \\
\hline \multicolumn{3}{|l|}{ AGE } \\
\hline$<50$ & 23 & 57.5 \\
\hline$\geq 50$ & 17 & 42.5 \\
\hline \multicolumn{3}{|l|}{ SEX } \\
\hline $\mathrm{F}$ & 13 & 32.5 \\
\hline $\mathrm{M}$ & 27 & 67.5 \\
\hline \multicolumn{3}{|l|}{ ECOG PS } \\
\hline 1 & 24 & 60 \\
\hline 2 & 16 & 40 \\
\hline \multicolumn{3}{|l|}{ TUMOR LOCATION } \\
\hline Parietal & 24 & 60 \\
\hline Frontal & 14 & 35 \\
\hline Temporal & 10 & 25 \\
\hline Occipital & 4 & 10 \\
\hline Brainstem & 1 & 2.5 \\
\hline Cerebellar & 1 & 2.5 \\
\hline \multicolumn{3}{|l|}{ HISTOPATHOLOGY } \\
\hline GBM & 30 & 75.0 \\
\hline Astrocytoma GIII & 9 & 22.5 \\
\hline Gliosarcoma (GIV) & 1 & 2.5 \\
\hline \multicolumn{3}{|l|}{ TYPE OF SURGERY } \\
\hline Maximal safe resection & 25 & 62.5 \\
\hline Complete resection & 11 & 27.5 \\
\hline Stereotactic biopsy & 4 & 10.0 \\
\hline \multicolumn{3}{|c|}{ PREOPERATIVE SIZE } \\
\hline$<5$ & 17 & 42.5 \\
\hline$>=5$ & 23 & 57.5 \\
\hline \multicolumn{3}{|c|}{ POSTOPERATIVE SIZE } \\
\hline$<5$ & 16 & 55.2 \\
\hline$>=5$ & 13 & 44.8 \\
\hline
\end{tabular}

Table 1: Patients and disease characteristics.

Adjuvant temozolomide was given to 25 GBM patients $(62.5 \%)$; twenty patients have completed their adjuvant 6 cycles without the need for dose modification. On the other hand, five GBM patients did not receive adjuvant temozolomide due to advanced renal, hepatic impairment, and or intolerance to the concurrent temozolomide. Adjuvant temozolomide was well tolerated, although, vomiting, deterioration in ECOG PS, and DVT were developed in $14(35 \%), 4(10 \%)$, and one $(2.5 \%)$ patients respectively.

Complete remission (CR) was developed in $16(40 \%)$ patients (11 patients were having no residual after surgery). Partial response (PR) was reported in 13 patients $(32.5 \%)$, and 11 patients $(27.5 \%)$ had stable disease (SD).

At a median follow up period of 12.5 months (range: 3-40);26 patients (65\%) died because of the disease, while 14 cases $(35 \%)$ are still alive. 7 patients $(17.5 \%)$ still in CR, 5 patients $(12.5 \%)$ had PR, and 28 patients (70\%) developed disease progression. among the 28 patients $(70 \%)$ who developed disease progression, the infield recurrence was observed in 24 patients $(86 \%)$, while two patients $(7 \%)$ developed marginal recurrence, and two patients (7\%) developed out-offield recurrence.

The estimated median PFS was 11.7 months; while the estimated PFS at 12 and 24 months were $46.8 \%$ and $32 \%$ respectively (Figure. 1). Multivariate analysis revealed that ECOG PS:2, temporal tumor location, and presence of behavior changes significantly decrease the PFS (Table 2).

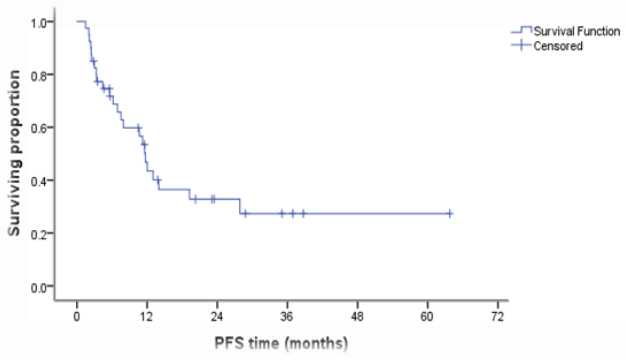

Fig 1: progression-free survival curve.

The estimated median OS was 14.31 months, and at 12 and 24 months were $62.6 \%$ and $35 \%$ respectively (Figure. 2). Multivariate analysis revealed that patients with ECOG PS:2 had worse OS than ECOG PS: 1 with HR $=8.21(95 \%$ CI: $3.091-21.839)$ and pvalue $<0.001$ (table 3 ).

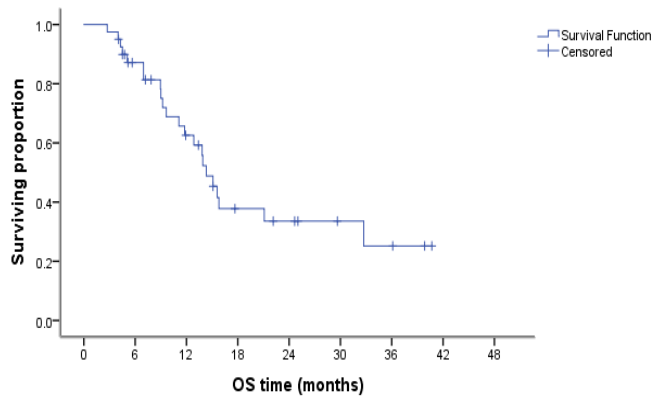

Fig 2: overall survival curve. 


\begin{tabular}{lllllll}
\hline & $\begin{array}{l}\text { Beta } \\
\text { coefficient }\end{array}$ & SD & p-value & HR & \multicolumn{2}{c}{ 95\% CI for HR } \\
\hline ECOG PS (2 versus 1) & 1.691 & 0.51 & 0.001 & 5.42 & 1.968 & 14.963 \\
\hline VOMITING (yes versus no) & 1.792 & 0.69 & 0.01 & 6.00 & 1.541 & 23.401 \\
\hline BEHAVIOR CHANGES (yes versus no) & 1.914 & 1.02 & 0.061 & 6.78 & 0.913 & 50.393 \\
\hline TEMPORAL LOCATION versus others) & 1.504 & 0.516 & 0.004 & 4.49 & 1.636 & 12.367 \\
\hline
\end{tabular}

Table 2: Multivariate analysis regarding PFS.

\begin{tabular}{lllllll}
\hline & $\begin{array}{l}\text { Beta } \\
\text { coefficients }\end{array}$ & SD & p-value & HR & \multicolumn{2}{c}{ 95.0\% CI for HR } \\
\cline { 5 - 7 } & 2.106 & 0.499 & $<0.001$ & 8.216 & 3.091 & 21.839 \\
\hline
\end{tabular}

Table 3: Multivariate Analysis regarding OS.

\section{DISCUSSION}

The most common type of HGGs is GBM that represents between $70-75 \%$. In this study, GBM patients were $(75 \%)$ and anaplastic astrocytoma was $(22.5 \%)$. This distribution of patients is compatible with the international similar trials ${ }^{1,12,13}$ where GBM represents about $80 \%$ of patients.

Radiotherapy is an essential part in the management of HGG patients, among factors that could affect the choice of radiotherapy dose and fractionation is the age and performance status of the patient according to the current NCCN recommendations; due to its effect on patient survival ${ }^{12}$, in this study PS was having a significant influence on survival, however, age didn't show the same effect on survival that could be explained by the low number of included patients.

Although the temporal lobe tumor location was less frequently appeared in our study (5\%), it was associated with worse outcome compared to parietal $(60 \%)$ and frontal $(35 \%)$ locations with $\mathrm{HR}=4.49$ (Pvalue $=0.004)$; while in the other published studies, the tumor locations involved by the disease are variable with no comments or significant relationship between location and response or survival. ${ }^{13}$

The long-term oncological outcome of HGGs is still dismal so that the quality of life is a very important consideration for patients treated with radiation therapy which is associated with acute and late neurotoxicities that are correlated with larger treatment volumes. Many oncology groups and centers like EORTC and Adult brain tumor consortium (ABTC) have adopted smaller radiotherapy margins for HGGs to reduce these toxicities and improve the quality of life, Furthermore; improved image quality by using MRI/CT fused images in delineation and using IMRT as advanced radiotherapy techniques both helping in better identification of target volumes and the critical organs, and reducing the dose to normal brain tissues. ${ }^{4,5,9}$
Excluding the edema from the treatment volumes in our study resulted in reducing median CTV and PTV volumes to $259.8 \mathrm{~cm}^{3}$ and $348 \mathrm{~cm}^{3}$ respectively which is comparable with the Kosztyla et al trial that used the same target volume delineation concept'; and reported median CTV and PTV was $249 \mathrm{~cm}^{3}$ and $369 \mathrm{~cm}^{3}$ respectively ${ }^{14}$, subsequently, no any serious toxicity during concurrent radio-chemotherapy and adjuvant temozolomide has been reported in our study as well as any treatment-related deaths that is also consistent with results of the other trials. ${ }^{15-17}$

The logic question raised after using limited CTV along with the IMRT technique is, does this technique increase the probability of marginal recurrence or not? Chang et al published a series showed that $90 \%$ of patients failed in central and in-field location, and found no statistical correlation between the location of the recurrent tumor and edema volumes and concluded that no differences were observed in the patterns of relapse between the RTOG two-phase (including all edema) and the EORTC single-phase (excluding edema) techniques ${ }^{7}$. Another study by Zhou et al; treat 54 HGG patients with limited CTV reported in-field recurrence in $77.8 \%$ of patients, marginal recurrence in $3.7 \%$, and distant recurrence in $3.7 \% .{ }^{18}$

On the other hand, trials used wider margins (RTOG guidelines) reported comparable in-field recurrence rate, as the study by Lee et al that reported $89 \%$ infield recurrence ${ }^{19}$. Another study by Brandes et al reported $21.5 \%$ out of field recurrence, and $6.3 \%$ recurrences at the margin, despite including the peri-tumoral edema in the treatment volumes ${ }^{20}$. Even after dose escalation up to $90 \mathrm{~Gy}$, still $91 \%$ of failure located within the treatment field as reported by Chan et al ${ }^{21}$. The reported patterns of failure in our study are also comparable with the results of the studies mentioned above where $86 \%$ of recurrences were infield, $7 \%$ at the margin, and $7 \%$ were out-field. 
Molecular classification of patients with HGGs is currently recommended as initial assessment with at least IDH and MGMT to predict survival and response to the alkylating agents (Temozolomide) ${ }^{22}$; despite that, concurrent use of temozolomide with radiotherapy is still recommended for HGG patients regardless the IDH and MGMT status; although the marginal benefit for patients with MGMT demethylation ${ }^{23}$; unfortunately these molecular tests are not available at our institution. In this study, $88 \%$ of patients completed the concurrent temozolomide along the whole course of radiotherapy and $80 \%$ of GBM patients received 6 months of adjuvant temozolomide which is almost similar to the results published by other authors, where $83.5 \%-86.6 \%$ of patients completed the temozolomide course ${ }^{1,16}$.

As mentioned before survival of $\mathrm{HGG}$ patients is still dismal, with median survival around one year and a minority of patients alive after 2 years. ${ }^{24,25}$ The median PFS and OS in our study were 11.71 months and 14.31 months respectively and one year PFS and OS were $46.8 \%$ and $62.6 \%$ respectively which like what is reported by the other studies..$^{1,3,9,18}$

\section{CONCLUSION}

In this study, the majority of relapses occurred at the original tumor location and only a few relapses occurred at the margin or outside the PTV; despite the relatively reduced margins around the gross residual tumor, that suggesting the efficacy and safety of using the IMRT technique with reduced CTV for management of HGG patients in our daily practice, especially in the view of good tolerability, PFS and OS, however, phase 3 randomized studies are needed to confirm this conclusion.

\section{REFERENCES}

1. Stupp R, Mason WP, van den Bent MJ, et al: Radiotherapy plus Concomitant and Adjuvant Temozolomide for Glioblastoma. $N$ Engl J Med. 2005; 352:987-96.

2. Joseph B, Malavade V, Nisarga V M, et al: Target Delineation in High-grade Glioma Do CTV Margins Make a Difference? ESTRO-ACROP versus RTOG Guidelines a Comparative Dosimetric Study. Advances in Modern Oncology Research. 2018; 04, 06.

3. Gilbert MR, Wang M, Aldape KD, et al: DoseDense Temozolomide for Newly Diagnosed Glioblastoma: A Randomized Phase III Clinical Trial. J Clin Oncol. 2013; 31:4085-91.

4. Dhermain F. Radiotherapy of high-grade gliomas: current standards and new concepts, innovations in imaging and radiotherapy, and new therapeutic approaches. Chin J Cancer. 2014; 33:16-24.

5. Gebhardt BJ, Dobelbower MC, Ennis WH, et al: Patterns of failure for glioblastoma multiforme following limited-margin radiation and concurrent temozolomide. Radiation Oncology. 2014; 9:130

6. Minniti G, Amelio D, Amichetti M, et al. Patterns of failure and comparison of different target volume delineations in patients with glioblastoma treated with conformal radiotherapy plus concomitant and adjuvant temozolomide. Radiother Oncol. 2010; 97: 377-81.

7. Chang EL, Akyurek S, Avalos T, et al: Evaluation of peritumoral edema in the delineation of radiotherapy clinical target volumes for glioblastoma. Int J Radiat Oncol Biol Phys. 2007; 68(1):144-150.

8. Milano MT, Okunieff P, Donatello RS, et al. Patterns and timing of recurrence after temozolomide-based chemoradiation for glioblastoma. Int J Radiat Oncol Biol Phys. 2010; 8:1147-55

9. Guram K, Smith M, Ginader T, et al: Using Smaller Than-Standard Radiation Treatment Margins Does Not Change Survival Outcomes in Patients with High-Grade Gliomas. Practical Radiation Oncology. 2019; 9, 16-23.

10. Mishra R, Mishra H, Mandal A, et al: Dosimetric Comparison of IMRT and Rapid ARC for High Grade Gliomas. Ann. Int. Med. Den. Res. 2019; 5(5): 1-3.

11. Wen PY, Macdonald DR, Reardon DA, et al. Updated response assessment criteria for highgrade gliomas: Response Assessment in NeuroOncology Working Group. J Clin Oncol. 2010;28:1963-72

12. Thumma SR, Fairbanks RK, Lamoreaux WT, et al: Effect of pretreatment clinical factors on overall survival in glioblastoma multiforme: a Surveillance Epidemiology. World Journal of Surgical Oncology. 2012; 10:75

13. Briere TM, McAleer MF, Levy et al. Sparing of normal tissues with volumetric arc radiation therapy for glioblastoma: single institution clinical experience. Radiation Oncology. 2017; 12:79.

14. Kosztyla R, Chan EK, Hsu F, et al: High-Grade Glioma Radiation Therapy Volumes And Patterns Of Failure Obtained From Magnetic Resonance Imaging And 18F-FDOBA Positron Emission Tomography Delineations From Multiple Observers. Int J Radiation Oncol Biol Phys. 2013; 87(5 ):1100-6

15. Hau P, Koch D, Hundsberger T et al: Safety and feasibility of long-term temozolomide treatment in patients with high-grade glioma. Neurology. 2007; 68:688-90

16. Khasraw M, Bell D, Wheeler H: Long-term use of temozolomide: could you use temozolomide safely for life in gliomas?. J Clin Neurosci. 2009; 16:854-5

17. Seiz M, KraVt U, Freyschlag CF, et al. Long-term adjuvant administration of temozolomide in patients with glioblastoma multiforme: experience of a single institution. J Cancer Res Clin Oncol. 2010; 136:1691-5

18. Zhou X, Liao X, Zhang B, et al: Recurrence patterns in patients with high-grade glioma following temozolomide- based chemoradiotherapy. Molecular And Clinical Oncology; 2016; 5: 289-94.

19. Lee SW, Fraass BA, Marsh LH, et al: Patterns of failure following high-dose 3-d conformal radiotherapy for high-grade astrocytomas: a quantitative dosimetric study. Int $J$ Radiat Oncol Biol Phys. 1999; 43(1):79-88. 
20. Brandes AA, Tosoni A, Franceschi E, et al: Recurrence pattern after temozolomide concomitant with and adjuvant to radiotherapy in newly diagnosed patients with glioblastoma: correlation with MGMT promoter methylation status. J Clin Oncol. 2009; 27(8):1275-9.

21. Chan JL, Lee SW, Fraass BA, et al: Survival and failure patterns of high-grade gliomas after three dimensional conformal radiotherapy. $J$ Clin Oncol. 2002; 20:1635-42

22. Louis DN, Ohgaki H, Wiestler OD, et al. WHO Classification of Tumors of the Central Nervous System. 2016; IARC, Lyon: 4th ed.

23. Hegi ME, Diserens AC, Gorlia T, et al. MGMT gene silencing and benefit from temozolomide in glioblastoma. N Engl J Med. 2005; 352:997.

24. Wen PY, Kesari S. Malignant gliomas in adults. $N$ Engl J Med. 2008;359:492-507.

25. DeAngelis LM. Brain tumors. $N$ Engl J Med; 2001;344:114-23. 